Ann. Biol. anim. Bioch. Biophys., I975, 15 (3), 569-582.

\title{
EFFET DE L'INSULINE \\ SUR LA COMPOSITION CORPORELLE ET LES TENEURS EN ACIDES AMINÉS LIBRES DU SANG, DU FOIE ET DU MUSCLE DU RAT EN CROISSANCE SOUMIS A UNE RESTRICTION ÉNERGÉTIQUE
}

\author{
J. GRIZARD, J. PRUGNAUD, M. ARNAL et R. PION \\ avec la collaboration technique de Françoise Barré et Marie Claude ValtuY \\ Laboratoire d'Étude du Métabolisme azoté, \\ Centre de Recherches de Clermont-Ferrand, I. N.R. A., \\ Theix, Saint Genès Champanelle, 63110 Beaumont
}

RÉSUMÉ

L'expérience réalisée avait pour objet de préciser dans quelle mesure l'insuline pouvait modifier l'utilisation de l'azote et de l'énergie chez le Rat en croissance, nourri en 6 repas égaux par jour et soumis à une restriction purement énergétique.

Dans ce but, des rats mâles de souche "Wistar " pesant $90 \mathrm{~g}$ ont été répartis en 3 lots : un lot A témoin recevant un régime équilibré à base de farine de hareng de Norvège (à II,7 p. Ioo de matières azotées) et deux lots $\mathrm{B}$ et $\mathrm{C}$ recevant un régime riche en protéines (à I $9,6 \mathrm{p}$. Ioo de matières azotées). Pour une consommation limitée à 60 p. Ioo de celle des animaux du lot témoin, les animaux des lots $B$ et $C$ ingèrent autant de protéines, de vitamines, de minéraux, mais seulement 60 p. roo de l'énergie. Les animaux du lot C reçoivent journellement 0,5 à I UI d'insuline, en injection sous-cutanée.

La composition corporelle et les teneurs en acides aminés libres du sang, du foie et du muscle sont déterminées sur chaque lot, à l'abattage, lorsque les animaux ont atteint un poids moyen de $150 \mathrm{~g}$.

La restriction énergétique ralentit la croissance (57 p. Ioo), diminue l'efficacité alimentaire apparente de l'azote et de l'énergie et l'adiposité du corps des animaux. Le traitement de ces animaux par l'insuline améliore la croissance, les rétentions azotées et énergétiques mais ne modifie pas la composition corporelle.

La restriction énergétique s'accompagne d'une diminution presque générale des teneurs en acides aminés libres non indispensables et d'une accumulation de la thréonine libre dans le sang, le foie et le muscle ; les teneurs de la plupart des acides aminés libres indispensables augmentent légèrement dans le muscle et diminuent dans le foie. Par rapport aux animaux dont l'alimentation énergétique est limitée, le traitement par l'insuline accentue la baisse des teneurs en acides aminés libres non indispensables dans le sang et le muscle, diminue les teneurs de la plupart des acides aminés libres indispensables dans le muscle et augmente les teneurs de la plupart de ces derniers dans le foie. 


\section{INTRODUCTION}

Malgré l'abondance des travaux concernant le jeûne ou la réduction globale des apports alimentaires, la restriction purement énergétique avec maintien du niveau des autres constituants du régime n'a fait l'objet que de peu d'études.

La mise au point de CAHIL et al., (I972) montre que le besoin énergétique est prioritaire car l'animal déclanche progressivement au cours du jeûne une série de processus métaboliques tendant à maintenir un apport suffisant de calories pour permettre le renouvellement de ses structures et sa survie. Bien que la principale réserve énergétique soit constituée par les graisses, 1'homéostasie du glucose est surtout assurée, au cours du jeûne, par la néoglucogénèse hépatique ; en effet, les animaux ne sont pas capables de synthétiser des précurseurs glucogéniques à partir de l'acétate dérivant des acides gras et les réserves de glycogène sont faibles. Les précurseurs sont donc pour une grande part les acides aminés provenant du catabolisme des protéines, puis le pyruvate et le lactate recyclés et enfin le glycérol.

Dans le cas de restrictions purement énergétiques chez le Rat en croissance, l'efficacité alimentaire apparente de l'azote est diminuée (RÉRAT et DESMOULIN, I970), la synthèse des ARN et des protéines musculaires réduite (DURAND, FauconnEaU et PENot, I967) et le catabolisme de la lysine augmenté (ARnaL, Fauconneau et PECH I972). Tout se passe comme si une partie des composés azotés était catabolisée et utilisée à des fins énergétiques. Ceci semble lié à une modification de l'équilibre hormonal, en particulier une réduction de la sécrétion d'insuline puisque cette hormone stimule le passage des acides aminés dans le muscle, la synthèse protéique (MANCHESTER, I970) et réduit le catabolisme protéique dans le foie (MortimorE et Mondon, I970).

C'est pourquoi le présent travail a pour but de préciser si le traitement par l'insuline est capable de modifier l'utilisation de l'azote et de l'énergie, en particulier au niveau des teneurs en acides aminés libres du foie, du sang et du muscle chez le Rat en croissance nourri par repas soumis à une carence purement énergétique.

\section{MATÉRIEL E'T MÉTHODES}

$$
\text { I. - Animaux d'expérience }
$$

Des rats mâles de souche "Wistar " sont élevés en cages individuelles dans des locaux éclairés de 22 heures le soir à Io heures le matin, maintenus à $22^{\circ} \mathrm{C}$ et de degré hygrométrique contrôlé $(60 \mathrm{p}$. I00). Ils sont sevrés vers $40 \mathrm{~g}$ et nourris d'un régime témoin distribué en 6 repas égaux à Io, I 4, I 8, 22, 2 et 6 heures. Lorsque leur poids vif est voisin de $90 \mathrm{~g}$, les animaux sont répartis en 3 lots identiques $\mathrm{A}, \mathrm{B}$ et $\mathrm{C}$; les animaux du lot $\mathrm{A}$ sont maintenus au régime témoin tandis que les animaux des lots $B$ et $C$ reçoivent un régime riche en protéines. La quantité de nourriture distribuée à ces derniers est progressivement limitée pour atteindre au bout de 3 jours, 60 p. Ioo de celle des animaux du lot $\mathrm{A}$.

Lorsque les animaux du lot $\mathrm{C}$ ont atteint le poids moyen de $\mathrm{I}$ oo g, des injections sous-cutanées d'insuline de bœuf (Novo ultralente) sont faites tous les jours entre I 2 et 13 heures; la dose diluée dans $0,5 \mathrm{ml}$ de sérum physiologique stérile passe progressivement de 0,5 à I UI/rat au dixième jour du traitement. Les animaux des lots A et B reçoivent des injections de sérum physiologique, effectuées dans les mêmes conditions.

Lorsqu'ils ont atteint un poids moyen de I $_{5} \mathrm{~g}$, les animaux d'un même lot sont sacrifiés le 
même jour à 15 heures par égorgement sans anesthésie. I a plus grande partie du sang est recueillie dans de l'éthanol $95^{\circ}$ refroidi pour le dosage des acides aminés, et une petite fraction est recueillie sur héparine pour la séparation du plasma. Le foie, les muscles des pattes postérieures sont rapidement prélevés, congelés dans l'azote liquide et conservés à -- $20^{\circ} \mathrm{C}$ sous sachets en plastique.

$$
\text { 2. - Régimes (tabl. 1) }
$$

Ce sont des régimes semi-synthétiques à base de farine de hareng de Norvège supplémentée en méthionine, phénylalanine et tryptophane. Les vitamines sont apportées dans de l'huile de maïs, de l'amidon et de l'eau selon la méthode et les quantités utilisées par Pawlak et Pios (I968 $a$ et $b$ ) et les minéraux sous forme d'un mélange dont la composition est indiquée dans le tableau 2. L'équilibre énergétique est assuré par de l'amidon de blé et de l'huile d'arachide.

TABLEAU I

Composition des régimes ( $\mathrm{g} / \mathrm{kg}$ de matière sèche)

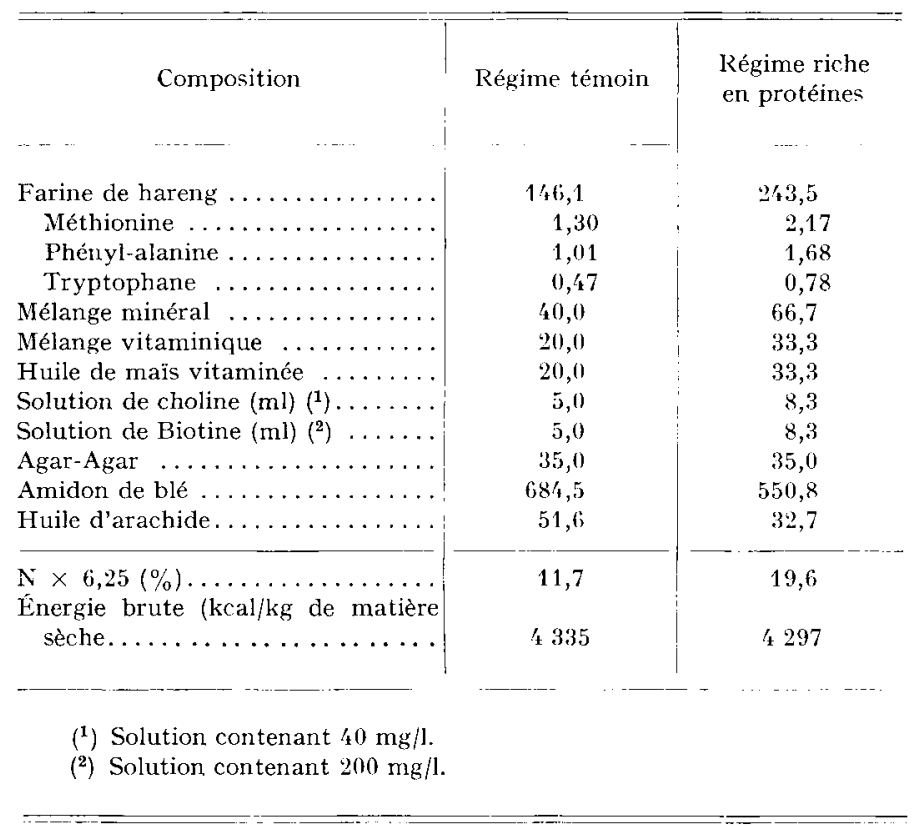

TABLEAU 2

Composition du complément minéral ajouté au régime témoin (par $\mathrm{kg}$ de matière sèche de régime)

\begin{tabular}{|c|c|c|}
\hline $\mathrm{PO}_{4} \mathrm{HCa}, 2 \mathrm{H}_{2} \mathrm{O} \ldots \ldots \ldots \ldots \ldots \ldots \ldots \ldots \ldots \ldots \ldots \ldots \ldots \ldots \ldots \ldots$ & \multicolumn{2}{|c|}{$32,31 \mathrm{~g}$} \\
\hline 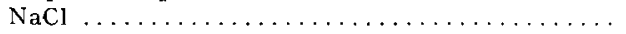 & & \\
\hline 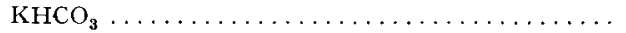 & & \\
\hline $\mathrm{CO}_{3} \mathrm{Mg} \ldots$. & & \\
\hline 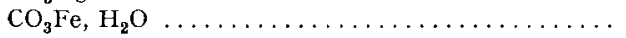 & 155 & $\mathrm{mg}$ \\
\hline 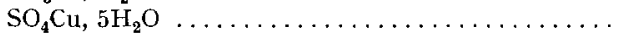 & 42 & $\mathrm{mg}$ \\
\hline $\mathrm{CO}_{3} \mathrm{Mn} \ldots \ldots \ldots \ldots \ldots \ldots \ldots \ldots \ldots$ & 90 & $\mathrm{mg}$ \\
\hline 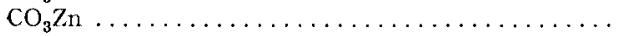 & 62 & $\mathrm{mg}$ \\
\hline $\mathrm{FNa}, \ldots \ldots \ldots \ldots \ldots \ldots \ldots \ldots \ldots \ldots \ldots \ldots$ & 12 & $\mathrm{mg}$ \\
\hline I K $\ldots$ & 281 & $\mu \mathrm{g}$ \\
\hline $2 \mathrm{CoCO}_{3} 3 \mathrm{Ca}(\mathrm{OH})_{2}$ & 188 & $\mu g$ \\
\hline
\end{tabular}


La composition du régime riche en protéines est telle que les animaux qui le consomment et dont l'ingestion est limitée à $60 \mathrm{p}$. 100 de celle des animaux témoins ingèrent autant de protéines, de minéraux et de vitamines que ccs derniers mats seulement $60 \mathrm{p}$. Ioo de l'énergie.

\section{3. - Mode d'alimentation par repas contrôlés}

La nourriture, semi-liquide, placée le matin dans des seringues de $5^{\circ} \mathrm{ml}$ est poussée à chaque repas dans des ramequins contenus dans les cages, grâce à des moteurs entraînant les pistons. En commandant la mise en marche et la durée du fonctionnement des moteurs, deux minuteries déterminent la fréquence des repas et la quantité approximative de nourriture journellement distribuée. Lorsque les animaux sont habitués, ils consomment chaque repas, sans refus, en $5 \mathrm{mn}$ environ. La quantité de nourriture consommée est estimée par pesée des seringues; la matière sèche de l'aliment distribué est mesurée sur des rations fictives clistribuées par 2 seringues témoins.

Les animaux disposent d'eau à volonté.

\section{4. - Méthodes analytiques}

Tous les dosages ont été effectués dans les tissus cles animaux groupés par lot.

a) Dosage des acides aminés libres du sang.

Les acides aminés libres sont extraits à partir de 20 à $30 \mathrm{~g}$ de sang, par épuisements successifs à l'éthanol $82 \mathrm{p}$. I oo contenant 2 p. Ioo de thiodiglycol pour éviter l'oxydation de la méthionine. Après purification des extraits sur Amberlite I R I2O, les acides aminés sont séparés et dosés sur résine échangeuse d'ions au moyen d'un analyseur automatique (SPACKMAN, STEIN et Moore, 1958). Cette technique permet le dosage de la plupart des acides animés libres, à l'exception des plus acides non retenus (taurine); elle permet en particulier de séparer la citrulline de la proline et de l'acide glutamique, la sérine de la glutamine; en revanche, elle ne permet pas de séparer l'asparagine de la glutamine et les résultats concernant ces deux composés sont calculés en glutamine.

\section{b) Dosage des acides aminés libres du toie et du muscle.}

Ils sont dosés selon la technique précédente après extraction par épuisements successifs à l'acide trichloracétique ro p. roo et purification sur Dowex II. L'éluat purifié est d'autre part traité par la soude et le sulfate de sodium pour transformer la cystéine en cystine et éliminer l'interférence du glutathion lors de la chromatographie (Moore, Spackman et Stein, I958).

\section{c) Dosage du glucose, de l'urée et de l'insuline dans le sang.}

Le glucose est dosé selon la méthode à la glucose-oxydase (Hill et Kessler, 1961) et l'urée selon la méthode à la diacétyl monoxine (MICHEL, 197I) à l'aide d'autoanalyseurs Technicon. La concentration en insuline plasmatique est déterminée par radioimmunologie selon la méthode de Hales et Randle (1963).

\section{d) Détermination de la composition corporelle.}

Les corps des animaux, groupés par lot, privés de leur sang, du contenu digestif et ayant subi l'ablation du foie et d'une partie des muscles des pattes postérieures (4 à $5 \mathrm{~g}$ ) sont soumis à 2 broyages successifs ; le premier est effectué en présence d'azote liquide au moyen d'un broyeur type Stephan; le second est effectué au moyen d'un broyeur type Robocoupe. Après homogénéisation du broyat au Turmix, la teneur en azote est mesurée par la méthode Macrokjeldahl, la teneur en énergie est déterminée au moyen d'un calorimètre adiabatique et la tencur en cendres par passage au four à $600^{\circ} \mathrm{C}$.

La matière sèche des résidus d'extraction des acides aminés libres du sang permet une estimation de la matière sèche du sang. 


\section{RÉSULTATS}

\section{I. - Croissance et efficacité alimentaire apparente des régimes (tabl. 3)}

Une réduction de $40 \mathrm{p}$. Ioo de l'apport énergétique de la ration s'accompagne d'un ralentissement de 57 p. Ioo de la croissance; l'indice de consommation est augmenté de 40 p. Ioo et les coefficients d'efficacité protéique (CEP) et énergétique (CEE) sont respectivement diminués de 57 et 28 p. Ioo.

TABLEAU 3

Consommation, croissance et efjicacilé alimensuire

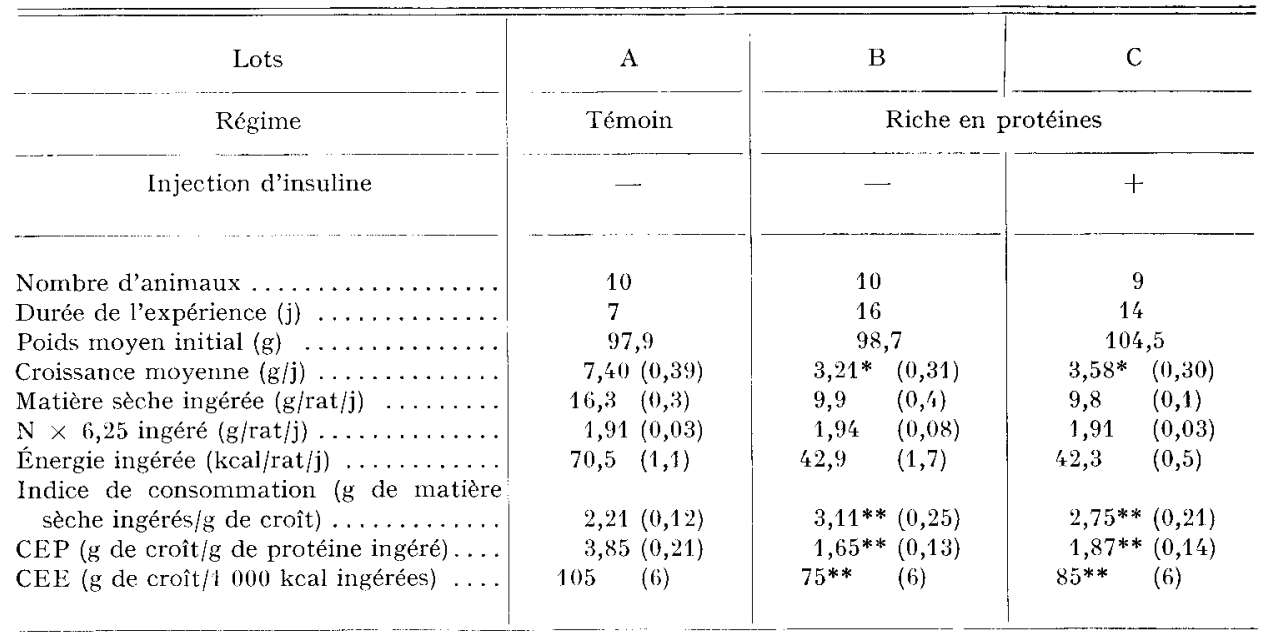

L'écart-type de la population est indiqué entre parenthèses.

La signification des différences entre les moyennes des lots $B$ et $C$ est analysée par le test $t^{\prime}>t$ (SNEDECOR, 1962) : * différents au seuil de 5 p. 100, ** différents au seuil de 2 p. 100.

Le traitement par l'insuline des animaux dont l'alimentation énergétique est limitée améliore significativement la croissance, l'indice de consommation (II,5 p. roo) et les coefficients d'efficacité protidique et énergétique (I3,3 p. Ioo).

\section{2. - Composition corporelle (tabl. 4)}

Les effets de la carence énergécique se manifestent surtout par une très nette diminution de l'adiposité, un enrichissement en protéines et en eau du corps des animaux, une augmentation de la teneur en matière sèche des résidus d'extraction des acides aminés libres du sang et une réduction du poids du foie. L'apport d'insuline ne semble pas avoir d'influence notable sur la composition corporelle des animaux dont l'alimentation énergétique est limitée. 
TABLEAU 4

Composition du corps des animaux à l'abattage.

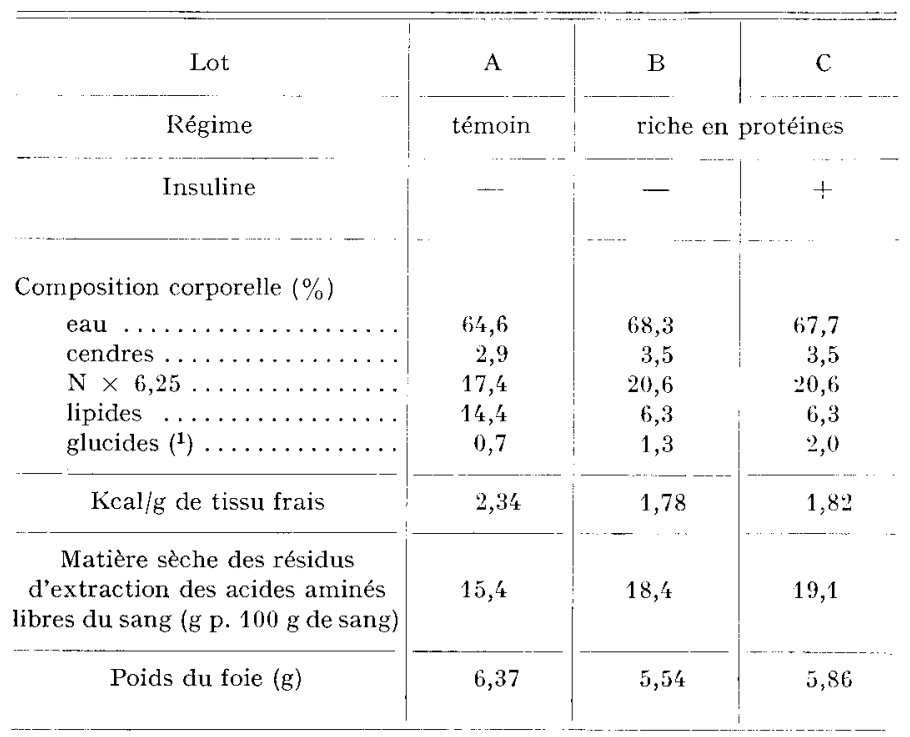

(1) Estimés à partir du bilan des masses et de l'énergie.

3. - Teneurs en acides aminés libres $d u$ sang, du foie et du muscle, glycémie, urémie et insulinémie (tab1. 5 et 6)

a) Teneurs en acides aminés libres du sang.

- Acides aminés libres non indispensables.

La restriction énergétique est marquée par une forte réduction de la teneur en acide glutamique ( 42 p. IOo) et une nette diminution des teneurs en alanine, acide aspartique, glycine (environ 30 p. roo), en citrulline, proline et sérine (environ 20 p. IOO) ; les teneurs en glutamine plus asparagine et ornithine ne varient pas. En dehors d'une augmentation des teneurs en ornithine et citrulline, le traitement hormonal provoque une réduction des teneurs en acides aminés libres non indispensables de l'ordre de $30 \mathrm{p}$. Ioo pour l'alanine et la proline et de I5 à $20 \mathrm{p}$. Ioo pour le mélange glutamine plus asparagine, la sérine, l'acide aspartique, la glycine et l'acide glutamique.

\section{- Acides aminés libres indispensables et semi-indispensables.}

La restriction énergétique entraîne surtout une forte accumulation de la thréonine ( $54 \mathrm{p}$. IOo), une légère augmentation de la teneur en leucine et une baisse des teneurs en lysine et phénylalanine.

Le traitement des animaux par l'insuline entraîne une augmentation de l'ordre de 5 p. Ioo des teneurs en acides aminés à chaîne ramifiée (isoleucine, leucine, valine) 


\section{TABLEAU 5}

Teneurs en acides aminés libres du sang, du foie et du muscle

( $\mathrm{mg}$ p. Ioo g de tissu frais) urémie ( $\mathrm{mg}$ p. Ioo $\mathrm{ml}$ de sang), glycémie ( $\mathrm{mg}$ p. Ioo $\mathrm{ml}$ de sang), insulinémie (uJ/ml de plasma)

\begin{tabular}{|c|c|c|c|c|c|c|c|c|c|}
\hline \multirow[b]{2}{*}{ Lots } & \multicolumn{3}{|c|}{ Sang } & \multicolumn{3}{|c|}{ Foie } & \multicolumn{3}{|c|}{ Muscle } \\
\hline & A & $\mathrm{B}$ & $\mathrm{C}$ & $\mathrm{A}$ & $\mathrm{B}$ & $\mathrm{C}$ & A & $\mathrm{B}$ & $\mathrm{C}$ \\
\hline Acide aspartique $\ldots \ldots \ldots$. & 0,7 & 0,5 & 0,4 & 10,5 & 6,6 & 6,4 & 5,5 & 3,9 & 3,5 \\
\hline Thréonine ........... & 2,0 & 5,1 & 4,4 & 3,8 & 5,5 & 6,5 & 6,9 & 15,0 & 12,6 \\
\hline Sérine $\ldots \ldots \ldots \ldots \ldots$ & 5,2 & 1,3 & 3,5 & 18,0 & 7,0 & 8,5 & 24,5 & 17,5 & 13,6 \\
\hline Glutamine (+ Asparagine) & 7,1 & 6,8 & 5,5 & 14,5 & 10,6 & 7,2 & 34,4 & 22,3 & 21,5 \\
\hline Acide glutamique $\ldots \ldots \ldots$ & 2,8 & 1,6 & 1,4 & 58,7 & 60,5 & 50,3 & 55,0 & 40,7 & 32,3 \\
\hline Proline $\ldots \ldots \ldots \ldots \ldots$ & 2,6 & 2,1 & 1,5 & 3,5 & 3,4 & 2,8 & 5,9 & 4,2 & 3,7 \\
\hline Citrulline . . . . . . . . . & 2,1 & 1,6 & 1,8 & trace & trace & trace & 6,1 & 4,5 & 1,9 \\
\hline Glycine . . . . . . . . . & 4,6 & 3,3 & 2,8 & 27,6 & 20,5 & 21,9 & 57,6 & 46,3 & 46,2 \\
\hline 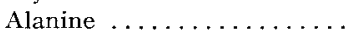 & 8,1 & 5,3 & 3,6 & 77,9 & 66,6 & 63,1 & 51,3 & 28,6 & 23,0 \\
\hline Valine. . . . . . $\ldots \ldots \ldots$ & 1,6 & 1,7 & 1,9 & 3,0 & 2,6 & 2,5 & 2,3 & 2,4 & 2,2 \\
\hline Cystine $\ldots \ldots \ldots \ldots \ldots$ & 0,4 & 0,4 & 0,3 & 1,3 & 1,7 & 2,3 & - & - & 一 \\
\hline Méthionine ( $\dashv$ Méthionine & & & & & & & & & \\
\hline sulfoxyde) $\ldots \ldots \ldots \ldots$ & 2,3 & 2,2 & 2,1 & 2,9 & 2,6 & 3,1 & 1,7 & 1,6 & 1,6 \\
\hline Isoleucine $\ldots \ldots \ldots \ldots \ldots$ & 0,9 & 0,8 & 1,0 & 2,4 & 1,8 & 2,1 & 1,2 & 1,3 & 1,0 \\
\hline Leucine $\ldots \ldots \ldots \ldots \ldots$ & 1,1 & 1,3 & 1,5 & 4,6 & 3,5 & 4,0 & 1,6 & 2,4 & 1,6 \\
\hline Tyrosine $\ldots \ldots \ldots \ldots \ldots$ & 1,0 & 1,0 & 0,9 & 2,1 & 1,4 & 1,1 & 1,8 & 2,0 & 1,5 \\
\hline Phényl-alanine $\ldots \ldots \ldots \ldots$ & 1,2 & 1,0 & 1,2 & 2,5 & 1,8 & 1,9 & 1,6 & 1,5 & 1,6 \\
\hline Ornithine $\ldots \ldots \ldots \ldots \ldots$ & 0,8 & 0,8 & 0,9 & 3,7 & 3,5 & 6,3 & 2,8 & 2,7 & 1,8 \\
\hline Lysine . . . . . . . . . . . & 9,9 & 8,3 & 7,5 & 10,8 & 7,3 & 8,3 & 44,1 & 40,6 & 27,6 \\
\hline Histidine $\ldots \ldots \ldots \ldots \ldots$ & 0,9 & 1,0 & 0,9 & 8,6 & 10,7 & 9,9 & 3,7 & 4,6 & 3,4 \\
\hline Arginine $\ldots \ldots \ldots \ldots \ldots$ & 3,2 & 3,6 & 3,2 & trace & trace & trace & 9,4 & 13,4 & 7,1 \\
\hline Ansérine. $\ldots \ldots \ldots \ldots \ldots$ & - & - & - & - & — & - & 27,8 & 43,3 & 48,4 \\
\hline 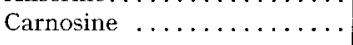 & - & - & - & 一 & 一 & - & 43,4 & 43,4 & 45,1 \\
\hline Somme des teneurs en AAI $\left({ }^{1}\right)$ & 24,7 & 26,3 & 24,9 & 42,1 & 39,0 & 41,6 & 74,4 & 84,9 & 60,2 \\
\hline Somme des teneurs en & & & & & & & & & \\
\hline $\begin{array}{l}\text { AANI }\left({ }^{2}\right) / \text { somme des te- } \\
\text { neurs en AAI }(\mathbf{1}) \ldots \ldots\end{array}$ & 1,38 & 1,01 & 0,86 & 5,09 & 4,58 & 4,00 & 4,22 & 3,03 & 4,05 \\
\hline Urée $\ldots \ldots \ldots \ldots \ldots \ldots \ldots$ & 9,3 & 18,4 & 21,6 & & & & & & \\
\hline Glucose $\ldots \ldots \ldots \ldots \ldots$ & 54,8 & 52,1 & 46,1 & & & & & & \\
\hline Insuline $\ldots \ldots \ldots \ldots \ldots$ & 51 & 80 & 82 & & & & & & \\
\hline
\end{tabular}

(1) AAI : Acides aminés indispensables + tyrosine et cystine.

(2) AANI : Acides aminés non indispensables. 
et une augmentation de I9 p. Ioo de la teneur en phénylalanine. Les proportions des différents acides aminés libres indispensables et semi-indispensables ne changent pas, sauf en ce qui concerne la thréonine dont la concentration est légèrement diminuée (tabl. 6).

\section{TABLEAU 6}

Teneurs en acides aminés libres indispensables et semi-indispensables

(p. roo de leur somme)

\begin{tabular}{|c|c|c|c|c|c|c|c|c|c|}
\hline \multirow[b]{2}{*}{ Lots } & \multicolumn{3}{|c|}{ Sang } & \multicolumn{3}{|c|}{ Foie } & \multicolumn{3}{|c|}{ Muscle } \\
\hline & A & B & $\mathrm{C}$ & A & $\mathrm{B}$ & $\mathrm{C}$ & $A$ & $\mathrm{~B}$ & $\mathrm{C}$ \\
\hline Thréonine ........ & 8,2 & 19,3 & 17,7 & 9,0 & 14,1 & 15,6 & 9,3 & 17,7 & 20,9 \\
\hline Valine $\ldots \ldots \ldots$. & 6,5 & 6,1 & 7,6 & 7,1 & 6,7 & 6,0 & 3,1 & 2,8 & 3,6 \\
\hline Cystine ........ & - & $\rightarrow$ & -- & 3,1 & 4,4 & 5,6 & - & - & 一 \\
\hline Méthionine ....... & 9,3 & 8,2 & 8,3 & 6,9 & 6,6 & 7,4 & 2,3 & 1,9 & 2,7 \\
\hline Isoleucine $\ldots \ldots \ldots$ & 3,7 & 3,0 & $f^{\prime}, 0$ & 5,7 & 4,6 & 5,0 & 1,6 & 1,5 & 1,7 \\
\hline Leucine . . . . . . . . & 4,5 & 4,9 & 6,0 & 10,9 & 9,0 & 9,6 & 2,1 & 2,8 & 2,7 \\
\hline Tyrosine ........ & 4,1 & 3,8 & 3,6 & 5,0 & 3,6 & 2,6 & 2,4 & 2,4 & 2,5 \\
\hline Phényl-alanine & 4,9 & 3,8 & 4,8 & 5,9 & s, 6 & 4,6 & 2,1 & 1,8 & 2,7 \\
\hline Lysine $\ldots \ldots \ldots$ & 40,4 & 31,5 & 30,1 & 25,7 & 18,8 & 19,9 & 59,3 & 47,9 & 45,8 \\
\hline Histidine . . . . . . & 3,7 & 3,8 & 3,6 & 20,5 & 27,5 & 23,7 & 5,0 & $5, \mathbf{4}$ & 5,6 \\
\hline Arğinine .... & 13,1 & 13,6 & 12,8 & 0 & 0 & 0 & 12,6 & 15,8 & 11,8 \\
\hline
\end{tabular}

b) Teneurs en acides aminés libres du foie.

La somme des teneurs en acides aminés libres est 4 à 5 fois plus élevée dans le foie et le muscle que dans le sang. Les foies ne contiennent ni citrulline, ni arginine libres et leurs teneurs en acides aminés à chaine ramifiée (isoleucine, leucine, valine) sont élevées.

\section{-- Acides aminés libres non indispensables.}

La restriction énergétique entraîne une réduction des teneurs en acides aminés libres non indispensables particulièrément importante pour la sérine (6I p. IOO), l'acide aspartique, le mélange glutamine plus asparagine et la glycine, plus faible pour l'alanine ( 14 p. roo); les teneurs en acide glutamique, proline et ornithine restent sensiblement constantes.

L'injection d'insuline aux animaux dont l'alimentation énergétique est limitée provoque une baisse de $32 \mathrm{p}$. Ioo de la teneur en glutamine plus asparagine et de I7 p. Ioo des teneurs en acide glutamique et proline et une augmentation des teneurs en ornithine ( $80 \mathrm{p}$. IOO) et sérine ( $22 \mathrm{p}$. IOO) ; les teneurs en acide aspartique, glycine et alanine restent pratiquement constantes.

- Acides aminés libres indispensables et semi-indispensables.

Ėn carence énergétique, les teneurs en thréonine, cystine et histidine sont nettement augmentées; les concentrations en tyrosine, lysine, phénylalanine, isoleucine et leucine diminuent de 25 à 33 p. Ioo et les teneurs en valine et méthionine d'environ Io p. IOO. 
Le traitement des animaux par l'insuline entraîne une augmentation de $37 \mathrm{p}$. Ioo de la teneur en cystine et d'environ ${ }_{5}$ p. Ioo des teneurs en thréonine, méthionine, leucine, lysine et isoleucine; seule la teneur en tyrosine diminue de façon importante (22 p. I00).

\section{c) Teneurs en acides aminés libres du muscle.}

- Acides aminés libres non indispensables.

Én carence énergétique, la teneur en ornithine reste constante alors que les teneurs des autres acides aminés libres non indispensables sont diminuées dans des proportions de $44 \mathrm{p}$. Ioo pour l'alanine, de $35 \mathrm{p}$. Ioo pour le mélange glutamine plus asparagine, de l'ordre de $27 \mathrm{p}$. Ioo pour la proline, l'acide aspartique, la sérine, l'acide glutamique et la citrulline et de $20 \mathrm{p}$. Ioo pour la glycine.

Le traitement par l'insuline diminue les teneurs de la plupart des acides aminés libres non indispensables dans des proportions allant de $30 \mathrm{p}$. Ioo pour l'ornithine à environ $20 \mathrm{p}$. Ioo pour la sérine, l'acide glutamique et l'alanine et environ $\mathrm{I} 2 \mathrm{p}$. Ioo pour la proline et l'acide aspartique ; seules les teneurs en glutamine plus asparagine, glycine et citrulline restent sensiblement constantes.

- Acides aminés libres indispensables et semi-indispensables.

En carence énergétique, les teneurs en acides aminés libres indispensables et semi-indispensables sont augmentées pour la thréonine (II7 p. IOo), la leucine, l'arginine et l'histidine et ne sont pas significativement modifiées pour la lysine, la méthionine, la phénylalanine, la valine, la tyrosine et l'isoleucine.

Le traitement hormonal entraîne une diminution des teneurs en arginine (47 p. IOo), leucine et lysine (environ 35 p. Ioo), histidine et tyrosine (27 p. IOo), isoleucine (I9 p. Ioo) et thréonine ( 16 p. I00).

\section{d) Glycémie, urémie et insulinémie.}

La glycémie des animaux soumis à la restriction énergétique est comparable à celle des animaux témoins; elle est légèrement plus faible chez les animaux traités par l'insuline. En revanche l'urémie et l'insulinémie, respectivement plus élevées de 97 et 57 p. Ioo chez l'animal dont 1'alimentation énergétique est limitée que chez l'animal témoin, ne sont pas modifiées par le traitement hormonal.

\section{DISCUSSION}

\section{I. - Choix d'une distribution de la nourriture en 6 repas égaux par jour}

La distribution de la nourriture en 6 repas évite l'influence des différences de rythme alimentaire. En effet, une restriction énergétique modifie fortement le rythme alimentaire du Rat nourri ad libitum qui consomme alors en un seul repas la ration journalière, ce qui risque d'accroître les décharges d'insuline ainsi que cela a été montré en réduisant le nombre des repas chez le Rat (WILEY et LEVEILIE, I970) ou chez 1'Homme (SchLIERF et RAETZER, I972) et fausse ainsi les comparaisons entre 
les lots. De plus, ce mode d'alimentation a permis d'éviter une augmentation de la consommation des animaux traités, consécutive à une stimulation de l'appétit par l'insuline. Ėn outre, le système automatique de distribution permet de diminuer les écarts entre les consommations des animaux d'un même lot.

\section{2. - Teneur en insuline plasmatique}

L'élévation de l'insulinémie chez les animaux soumis à la restriction énergétique peut s'expliquer vraisemblablement par une stimulation de la sécrétion par les acides aminés indispensables absorbés plus rapidement (FAJANs et al., I967) ou par les hormones intestinales (sécrétine, pancréozymine) sécrétées en plus grande quantité du fait de la teneur élevée en protéines du régime. Toutefois la portée de ce résultat est limitée compte tenu des modifications importantes que subit l'insulinémie au cours du nycthémère (GAGLIARDINo et HERNANDEZ, I97I).

L'injection d'insuline ne provoque pas d'augmentation de l'insuline circulante ; elle est sans doute rapidement dégradée par le foie car sa demi-vie n'est que de 4 à io mn (Cathelineau, Val,

\section{3. - Utilisation de l'azote 't de l'énergie}

La faible augmentation de la teneur en azote du corps des animaux et la très nette diminution du coefficient d'efficacité protidique associée à une baisse plus modérée du coefficient d'efficacité énergétique montrent que la fixation journalière d'azote est considérablement diminuée par la restriction énergétique : 1'animal utilise à des fins énergétiques une grande partie des protéines surajoutées au régime et ce résultat confirme les observations de Rúra'T et Desmoulin (I970) chez le Rat à différents âges et pour plusieurs niveaux de carence énergétique.

La restriction énergétique entraîne une baisse importante du rendement de fixation de l'énergie sans doute due en partie au coût énergétique de la dégradation des protéines dont l'importance est accrue ainsi que l'ont constaté Arnal, FauconNEAU et PECH (I972).

Le traitement par l'insuline des animaux soumis à la restriction énergétique provoque de meilleures rétentions azotées et énergétiques sans modification de la composition corporelle, contrairement à ce qui est observé chez le Rat normalement nourri (BEATON cité par SNipes, I968) chez qui un tel traitement entraîne une surcharge en lipides.

\section{4. - Teneurs en acides aminés libres du sang, au foic et du muscle}

\section{a) En carence énergétique.}

Les fortes diminutions des teneurs de la plupart des acides aminés libres non indispensables, en particulier des principaux acides aminés glucoformateurs (alanine, sérine, acide glutamique, glycine et acide aspartique), à la fois dans le sang, le foie et le muscle montrent que la restriction énergétique entraîne une augmentation importante du catabolisme de ces acides aminés et de la néoglucogénèse hépatique. Dans le muscle, la réduction des teneurs en alanine et glutamine plus asparagine libres, plus intense que la réduction des teneurs des autres acides aminés libres non indispensables, peut s'expliquer par un accroissement du passage de ces composés du muscle 
vers le sang, ainsi que l'ont constaté CAHIL et al., (I972) chez l'Homme soumis à un jeûne. $L_{e}$ fait que les teneurs en alanine et glutamine plus asparagine libres sanguines n'augmentent pas résulte probablement d'un accroissement de l'utilisation d'alanine et de glutamine respectivement par le foie et l'intestin (AIKAwA et al., I973).

Dans le foie, des diminutions des teneurs de la plupart des acides aminés libres indispensables résultent vraisemblablement à la fois d'une augmentation de leur catabolisme et d'un accroissement de l'anabolisme protéique hépatique. ArNaL, FAUCONNEAU et PECH (I972) ont en effet montré que la carence énergétique chez le Rat entraîne une augmentation des vitesses de synthèse et de dégradation des protéines dans l'ensemble du compartiment digestif.

Le doublement de l'urémie montre que l'accroissement du catabolisme des acides aminés dans le foie entraîne une augmentation de la production d'urée par cet organe qui est sans doute responsable de la diminution de la teneur en citrulline libre sanguine et musculaire.

L'augmentation des teneurs en thréonine, leucine, arginine et histidine libres dans le muscle et des teneurs en thréonine et leucine libres dans le sang est vraisemblablement due à un ralentissement de la synthèse et à un accroissement du catabolisme des protéines musculaires. Les augmentations trouvées paraissent plus faibles que les augmentations décrites chez 1'animal soumis à un jeûne, en particulier au niveau des acides aminés à chaine ramifiée (valine, leucine, isoleucine) qui sont peu catabolisés par le foie (MunRo, I970).

La diminution de la teneur en lysine libre sanguine résulte d'un accroissement du catabolisme de cet acide aminé puisque Arnal, Fauconneau et Pech (I972) ont montré qu'une carence énergétique de 50 p. Ioo chez le Rat augmentait considérablement le catabolisme de la lysine, car la quantité de radioactivité dégagée en 8 heures sous forme de $\mathrm{CO}_{2}$ à partir de lysine marquée injectée à l'animal était multipliée par 3. L'augmentation de la teneur en lysine libre du sang et du muscle trouvée par ces auteurs était probablement due à la richesse en lysine du régime qu'ils employaient. En revanche, la thréonine s'accumule dans tous les compartiments étudiés et elle paraît moins catabolisée que la 1ysine. Ce résultat est en accord avec les expériences faites par PAWLAK et PION (I968 $a$ et $b$ ) au cours desquelles la thréonine s'accumule plus rapidement que la lysine dans le sang et le muscle du Rat soumis à des régimes supplémentés par des doses croissantes de ces acides aminés.

Les modifications des teneurs en acides aminés libres des tissus chez le Rat dont l'alimentation énergétique est limitée semblent donc indiquer que l'adaptation de ce dernier est surtout marquée par un ralentissement de l'anabolisme protéique musculaire et une activation de l'utilisation des acides aminés par le foie.

\section{b) En carence énergétique après traitement par l'insuline.}

Les diminutions importantes des teneurs en acides aminés libres indispensables dans le muscle, associées à l'amélioration de la croissance sans diminution de la teneur en azote du corps des animaux montrent que le traitement par l'insuline provoque une augmentation de la synthèse et une réduction de la dégradation des protéines musculaires. Ce résultat confirme le fait que l'insuline stimule à la fois le transport dans le muscle et l'incorporation des acides aminés marqués dans les protéines (Woor et al., I f(6) $^{6}$; le fait que les teneurs en acides aninés libres indispensables 
n'augmentent pas dans le muscle résulte d'une stimulation plus intense de l'utilisation des acides aminés pour la synthèse protéique que du transport de ces composés (MANCHESTER, I970).

Le traitement par l'insuline n'entraîne pas de baisse notable des teneurs en acides aminés libres indispensables dans le sang, contrairement à ce qui a été observé chez 1'Homme (Zimmermann-Telschow, Bethge et Herberg, ig67 ; SWEndseid et al., I967), le Rat (FERnstrom et WurTman, I972) et le Mouton (CALL et al., I972). Ceci est dû au fait que l'effet de l'accroissement du passage des acides aminés indispensables du sang au muscle est masqué par un apport important à la suite du repas consommé I heure avant le sacrifice.

Dans le foie, l'augmentation de la plupart des teneurs en acides aminés libres indispensables résulte vraisemblablement à la fois d'une réduction de leur catabolisme et d'un ralentissement de l'anabolisme protéique hépatique.

La diminution de la plupart des teneurs en acides aminés libres non indispensables dans le sang et le muscle résulte de l'accroissement de l'anabolisme protéique musculaire. Le fait que les teneurs en acides aminés libres non indispensables sanguines subissent l'effet de la stimulation de la synthèse protéique alors que les teneurs en acides aminés libres indispensables ne changent pas est vraisemblablement dû au fait que les acides aminés non indispensables sont davantage catabolisés. Cette baisse des teneurs en acides aminés libres non indispensables dans le sang limite leur transport en particulier dans le foie, ce qui diminue sans doute le catabolisme de ces composés et l'intensité de la néoglucogénèse (CAHIL, et al., I972). De plus, l'insuline ralentit probablement les voies métaboliques de la néoglucogénèse puisque SLADEK (I97I) a constaté que l'hormone entraîne une réduction du rythme de conversion en glucose de l'alanine ${ }^{14} \mathrm{C}$ dans le foie perfusé de rat.

Bien que l'urémie reste constante, l'accumulation de l'ornithine dans le foie, l'augmentation des teneurs en ornithine et citrulline libres sanguines traduisent sans doute un ralentissement du fonctionnement du cycle de l'urée dans le foie consécutif à la diminution du catabolisme des acides aminés.

Le traitement par l'insuline semble donc induire un accroissement de l'anabolisme protéique musculaire ; 1'utilisation accrue des acides aminés se traduit par une baisse générale des teneurs en acides aminés libres dans le muscle mais n'affecte que les teneurs en acides aminés libres non indispensables dans le sang.

\section{CONCLUSION}

La réduction de l'apport énergétique de la ration avec maintien du niveau azoté dans le régime, entraîne chez le Rat en croissance une utilisation accrue des protéines à des fins énergétiques. Elle s'accompagne d'une réduction presque générale des teneurs en acides aminés libres non indispensables dans le sang, le foie et le muscle, une diminution de la plupart des teneurs en acides aminés libres indispensables dans le foie et une légère augmentation des teneurs de ces derniers dans le muscle. Ces modifications semblent traduire une stimulation de la néoglucogénèse et du catabolisme des acides aminés, un ralentissement de l'anabolisme protéique musculaire et une augnentation des vitesses de synthèse et de dégradation des protéines par le foie. 
Le traitement des animaux par l'insuline améliore les rétentions azotées et énergétiques. Il provoque une diminution de la plupart des teneurs en acides aminés libres non indispensables dans le sang et le muscle, une augmentation des teneurs de la plupart des acides aminés libres indispensables dans le foie et une réduction des teneurs de la plupart de ces derniers dans le muscle. Tout se passe comme si l'insuline stimulait la synthèse ou ralentissait le catabolisme des protéines musculaires et limitait l'intensité de la néoglucogenèse et le catabolisme des acides aminés. Toutefois l'animal semble encore utiliser une part importante des composés azotés à des fins énergétiques.

Reçu pour publication en tévrier 1975.

\section{REMERCIEMENTS}

Nous remercions vivement Monsieur J. Charrier, MM. P. Thivend et M. C. Michel qui ont bien voulu effectuer respectivement les dosages d'insuline, de glucose et d'urée.

\section{SUMMARY}

EFFECT OF INSULIN ON BODY COMPOSITION

AND FREE AMINO ACID LEVELS IN BLOOD, LIVER AND MUSCLE OF THE GROWING RAT SUBJECTED TO ENERGETIC RESTRICTION

The experiment is carried out to study the effect of insulin on nitrogen and energy utilization in growing rats subjected to restricted energy intake.

The animals are male rats of the Wistar strain, weighing go $g$ at the beginning of the experiment. They are fed every 4 hours with a meal of equal weight. Control group A is fed a balanced diet with II.7 p. Ioo crude protein ; 2 experimental groups, B and C, receive a high protein diet with I9.6 p. Ioo crude protein (table I). Their food intake is restricted to $60 \mathrm{p}$. roo of that of the controls. They receive, thus, every day the same amount of crude protein, mineral and vitamins as the control group, but only $60 \mathrm{p}$. Ioo of the energy intake. At approximately 12.30 p.m. every day, animals of group $\mathrm{C}$ are injected subcutaneously with insulin starting at 0.5 units, which is progressively increased to I unit per day. Animals of groups A and B are sham-injected with saline. When the subjects of the same group have a mean body weight of ${ }_{15} \mathrm{O} g$, they are all slaughtered at 3 p.m., i.e. I hour after the meal. Food efficiency, body composition, blood, liver and muscle free amino acid levels are recorded.

The results show that reducing the energy supply of the ration results in a decrease of the growth rate, nitrogen and energy efficiency and body fat deposition. Insulin injection improves growth rate, nitrogen and energy efficiency, but does not affect body composition (tables 3 and 4).

Reduction of energy supply in the ration results in a decrease of most of the non-essential free amino acids and a large increase of free threonine in the blood, liver and muscle. Most of the other essential free amino acids increase in muscle but decrease in liver. Insulin injection results in a decrease of non-essential free amino acids in blood and muscle; it increases most essential free amino acids in liver but decreases most essential free amino acids in muscle (tables 5 and 6).

\section{RÉFÉRENCES BIBLIOGRAPHIQUES}

Alkawa T., Matsutaka H., Yamanoto H., Okuda T., Ishlkawa L., Kawano T., Matsumura E., 1973. Glucogenesis and anino acid metabolism. Il. Inter-organal relations and roles of glutamine and alanine in the animo acid netabolism of fasted rats. J. Biochem., 74, Ioo3-10I7. 
Arnal M., Fauconneau G., Pech R., 1972. Synthèses protéiques in vivo dans divers tissus du rat en croissance soumis à une réduction de l'apport énergétique de la ration. Ann. Biol. anim. Bioch. Biophys., 12, $9 \mathrm{I}-108$.

Cahill G. F., Aoki T. T., Brennan M. F., Mijllek W. A., ig72. Insulin and muscle amino acid balance. Proc. Nutr. Soc., 31, 233-238.

Call J. L., Mitchel G. E., Ely D. G., Little C. O., Tucker R. E., ig72. Amino acids, volatile fatty acids and glucose in plasma in insulin-treated sheep. J. Anim. Sci., 34, 767-77r.

Cathelineau G., Valleron A. J., Canivet J., i97o. Vitesse de disparition dans le plasma de l'insuline exogène non marquée. Ann. Endocr., 31, $271-276$.

Durand G., Fauconneau G., Penot J., I967. Croissance des tissus du rat et réduction de l'apport énergétique de la ration; influence sur la teneur en acides nucléiques. Ann. Biol. anim. Bioch. Biophys., $7,145-155$.

Fajans S. S., Floyd J. C., KNopf R. F., Conn J. W., I967. Effect of amino acids and proteins on insulin secretion in man. Rec. Prog. Horm. Res., 23, 617-662.

Fernstrom J. D., Wurtman R. J., I972. Elevation of plasma tryptophan by insulin in rat. Metab., 21,337-342.

Gagliardino J. J., Hernandez R. E., 1971. Circadian variation of the serum glucose and immunoreactive insulin levels. Endocrinology, 88, I529-1531.

Hales C. N., Randle P. J., I963. Immunoassay of insulin with insulin-antibody precipitate. Bio-

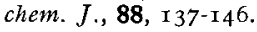

Hill J. B., Kessler G., I96I. An automated determination of glucose utilizing a glucose oxydaseperoxydase system. J. Lab. Clin. Med., 57, 970-980.

Manchester K. L., I970. The control by insulin of amino acid accumulation in muscle. Biochem. $J ., 117,457-465$.

Michel M. C., r97I. Analyse quantitative de quelques substances azotées et glucidiques en milieu biologique. Thèse Doctorat d'Université, Clermont-Ferrand.

Moore S., Spackman D. H., Stein W. H., I958. Chromatography of amino acids on sulfonated polystyrene resins. Analyt. Chem., 30, I I85-I I90.

Mortimore G. E., Mondon C. E., I970. Inhibition by insulin of valine turnover in liver. J. Biol. Chem., 245, 2375-2383.

Munro H. N., 1970. Free amino acid pools and their role in regulation In : Mammalian Protein Metabolism, Ed. Munro H. N., 4, 299-386. Academic Press. New York and London.

Pawlak M., Pion R., I968 a. Influence de la supplémentation des protéines du blé par des doses croissantes de lysine sur la teneur en acides aminés libres du sang et du muscle du rat en croissance. Ann. Biol. anim. Bioch. Biophys., 8, $517-530$.

Pawlak M., Pion R., r 968 b. Influence de la supplémentation des protéines du blé par des doses croissantes de thréonine sur les teneurs en acides aminés libres du sang total et du muscle du rat en croissance. C. R. Acad. Sc. Paris, 266, r993-I995.

Rérat A., Desmoulin B., I970. Influence d'une restriction alimentaire, énergétique et azotée sur la croissance et la composition corporelle du rat blanc. Ann. Zootech., 19, Io3-II 5.

Schlierf G., Raetzer H., r972. Diurnal patterns of blood sugar, plasma insulin, free fatty acid and triglyceride levels in normal subjects and in patients with type IV hyperlipoproteinemia and the effect of meal frequency. Nutr. Métab., 14, xI3-I 26.

SLADEK C. D., I97I. The relationship between the amino acid, glucose and insulin concentration in control of gluconeogenesis in perfused rat liver. Diss. Abstr. Int. B, 32, 3609 .

SNEDECOR G. W., I962. In statistical method applied to experiments in agriculture and biology, 5ed, Iowa State University Press. Ames, Iowa.

SNipes C. A., I968. Effects of growth hormone and insulin on amino acid and protein metabolism. Quart. Rev. Biol., 43, 127-147.

Spackman D. H., Stein W. H., Moore J., I958. Automatic recording apparatus for use in the chromatography of amino acids. Analyt. Chem., 30, I I90-I 206.

Swendeid M. E., Tutrle S. G., Drenick E. J., Jovien C. B., Massiey F. J., ig67. plasma amino acid response to glucose administration in various nutritive states. $A m$. J. Clin. Nutr., 31, $243-249$.

Wiley J. H., Leveille G. A., i 97o. Significance of insulin in the metabolic adaptation of rats to meal ingestion. J. Nutr., 100, 1073-1080.

Wool I. G., Stirewalt W. S., Kurihara K., Low R. B., Bailley P., Oyer D., ig68. Mode of action of insulin in the regulation of protein biosynthesis in muscle. Rec. Prog. Horm. Res., 24, I39-2 I3.

Zimmermann-Telschow H., Bethge H., Herberg L., I967. Changes in amino acids in plasma during the insulin stress test in man. Klin. Wochenschr., 45, 768-777. 